\title{
Variações da Dentogênese em Indivíduos do Grupo Pré-Histórico de Santana do Riacho, Lagoa Santa, Minas Gerais
}

\author{
Variations in Tooth Formation in Individuals from a Prehistorical Group \\ in Santana do Riacho, Lagoa Santa, Minas Gerais, Brazil
}

\author{
Sheila M. de Souza ${ }^{1}$
}

SOUZA, S. M. Variations in Tooth Formation in Individuals from a Prehistorical Group in Santana do Riacho, Lagoa Santa, Minas Gerais, Brazil. Cad. Saúde Públ., Rio de Janeiro, 9 (supplement 1): 96-98, 1993.

This article discusses variations in dentogenesis and their consequences when estimating age in archaeological samples. One example described here, taken from a sample of a Lagoa Santa primeval population found in the Santana do Riacho rock shelter in the state of Minas Gerais, Brazil, suggests a need for more research concerning patterns of dental growth and maturation in South American indigenous populations.

Key words: Paleodontology; Age Determination by Teeth; Indians; South America

A dentogênese e a osteogênese, sendo expressões de um processo multifatorial, relacionamse a fatores genéticos e ambientais, sendo de interesse médico, antropológico e forense. Proporcionam elementos à estimativa de idade biológica e refletem tanto condições hereditárias como o impacto de carências nutricionais e/ou outras formas de estresse sobre os indivíduos. Por tais razões, estudos sobre crescimento e maturação óssea e dentária estão entre os mais importantes para o estudo de amostras esqueletais humanas de procedência arqueológica (Johnston \& Zimmer, 1989). Tais pesquisas fornecem elementos para a comparação de grupos pré-históricos e populações indígenas atuais, do ponto de vista demográfico, genético ou das transformações da qualidade de vida e saúde, como nos estudos de tendência secular. No entanto, sendo processos independentes, nem sempre observa-se correspondência entre as cronologias da dentogênese e da osteogênese

\footnotetext{
${ }^{I}$ Departamento de Endemias Samuel Pessoa da Escola Nacional de Saúde Pública. Rua Leopoldo Bulhões 1480, Rio de Janeiro, RJ, 21041-210, Brasil.
}

num mesmo indivíduo (Hillson, 1990), o que acarreta contradições ou erros nas estimativas de idade biológica para fins legais ou arqueológicos.

Entre grupos indígenas norte-americanos, padrões peculiares de dentogênese, como a rizogênese mais acelerada, têm sido descritos (Ubelaker, 1978). Estes achados devem ser levados em consideração nos estudos de populações atuais ou pretéritas. Variações ao nível individual são muito comuns e, quando ocorrem ao nível populacional, podem ter significado biológico, exprimindo, por exemplo, variações genéticas. Um exemplo de variação deste tipo é encontrado entre os índios Arikara norteamericanos, cuja formação do terceiro molar, sendo mais precoce que o normal, leva a estimativas de idade dentária adiantadas em mais de 2 anos em relação à idade cronológica (Owsley \& Jantz, 1983). Com base nesta observação e no conhecimento de que as variações são mais freqüentes nos dentes anteriores e posteriores, este mesmo autor propõe que se utilize como base mais confiável a para estimativa de idade dentária os pré-molares.

Tal questão torna-se mais relevante se lem- 
brarmos que, de modo geral, a dentogênese é considerada menos sujeita a variações e, portanto, tida como um critério relativamente seguro para a estimativa de idade (Steele \& Bramblet, 1988). Desta forma, o estudo isolado da dentogênese em indivíduos ou grupos que apresentam variação em relação ao padrão dado como normal pode originar discrepâncias na estimativa de idade, com reflexos sobre a identificação individual e sobre as conclusões paleodemográficas e paleoepidemiológicas, por exemplo. É importante, deste modo, o registro de variações do desenvolvimento dentário, devendo sua recorrência ser investigada em grupos étnicos atuais ou pré-históricos.

Um cemitério arqueológico localizado e escavado pela equipe do arqueólogo André Prous, da Universidade Federal de Minas Gerais (UFMG), em um abrigo calcário de Santana do Riacho, Minas Gerais, Brasil, proporcionou cerca de 43 esqueletos humanos em estado variável de conservação, representando indivíduos de todas as faixas etárias e de ambos os sexos. O estudo osteológico desta amostra já investigou aspectos demográficos (Souza \& Alvim, s/d), dentários (Radichi, 1986; Souza, s/d) e patológicos (Souza, s/d). A antiguidade estimada para os sepultamentos foi estabelecida em cerca de 8.000 anos antes do presente (Prous, 1989). A morfologia esqueletal humana e o contexto arqueológico associado são compatíveis com os das chamadas populações primevas de Lagoa Santa (Alvim, 1977), formadas por coletores-caçadores pré-históricos que habitavam as regiões de savana de Minas Gerais.

Em exemplares de esqueletos de jovens e adultos de Santana do Riacho foram observadas variações na cronologia da rizogênese e da erupção dentária, cujo início é definido pelo aparecimento das criptas ao nível do plano ósseo superficial dos maxilares. Um estudo paleopatológico por Souza (s/d) descreveu a ausência de terceiro molar, que não é usual na população primeva de Lagoa Santa (Alvim, 1977), bem como o retardo na erupção do mesmo. O estudo radiológico conduzido por Raddichi (1986) mostrou que a não-erupção dos terceiros molares era explicada pelo estágio inicial de rizogênese em que os dentes se encontravam. Neste mesmo material, por outro lado, os dentes anteriores mostravam estágio avançado de rizogênese, observação coerente com o que descreve Ubelaker (1978) para grupos indígenas norte-americanos.

Em dois indivíduos cuja preservação permitiu uma análise mais detalhada, as estimativas de idade por critério de dentogênese e osteogênese levaram a resultados não-concordantes. $\mathrm{O}$ estágio de dentogênese, com os terceiros molares inclusos, em fase inicial da rizogênese, levou à estimativa de uma idade de 14 anos para os dois indivíduos. A análise da osteogênese, com base no estágio de maturação e fusão epifisária, na sinostose da sutura esfenobasilar, além de outros indicadores (Steele \& Bramblet, 1988), levou a estimativas de 18-20 anos (exemplar V A, feminino) e 20-25 anos (exemplar VI A, masculino), respectivamente.

A variação individual observada na formação do terceiro molar é freqüente e não chega a ser considerada patológica, tendo em vista os critérios de Johanson (appud Hillson, 1990). A observação desta variação confirma o que está dito em Hillson (1990), mostrando, mais uma vez, os riscos de se estimar a idade com base nesse dente. A presença desta variação pode, por outro lado, relacionar-se a um padrão genético ao nível populacional, devendo a mesma ser reconhecida para que as estimativas de idade feitas em amostras mal preservadas ou em esqueletos fragmentados não conduzam a desvios de grande magnitude.

Radichi (1986) sugeriu a etiologia ambiental para a aparente aceleração da dentogênese anterior nos indivíduos de Santana do Riacho. Esta resultaria de uma resposta da plasticidade fenotípica ao estímulo mecânico associado à abrasão dentária, que neste grupo é muito precoce e intensa, iniciando-se aos 2-3 anos de idade, e atinge as crianças principalmente na bateria labial. A importância da ação mecânica sobre a osteogênese é conhecida pela lei de Wolff (Wright \& Symmers, 1966). Da mesma forma, o papel da ação mecânica sobre a dentogênese parece refletir-se no processo contínuo de erupção tanto pelo crescimento da dentina como pela deposição aumentada de cemento dentário na raiz, esta última resultando da necessidade de manutenção do plano oclusal (Hilson, 1990).

Infelizmente, o estado de conservação da 
amostra de Santana do Riacho impede a verificação da existência de um padrão local variante de dentogênese. Contudo, há outras coleções de Lagoa Santa que podem ser estudadas do ponto de vista radiológico. As observações aqui descritas são importantes como forma de incentivar estudos de processos de maturação dentária tanto em coleções arqueológicas como entre grupos indígenas atuais, com vistas ao estabelecimento de padrões de dentogênese específicos para as populações da América do Sul.

\section{RESUMO}

SOUZA, S. M. Variações da Dentogênese em Indivíduos do Grupo Pré-Histórico de Santana do Riacho, Lagoa Santa, Minas Gerais. Cad. Saúde Públ., Rio de Janeiro, 9 (suplemento 1): 96-98, 1993.

A artigo discute as variações da dentogênese, e sua repercussão sobre a estimativa de idade em amostras arqueológicas. Um exemplo aqui descrito, proveniente da amostra da população primeva de Lagoa Santa encontrada no abrigo de Santana do Riacho, em Minas Gerais, Brasil, sugere a necessidade de mais estudos sobre padrões de crescimento e maturação dentária em populações indígenas da América do Sul.

Palavras-Chave: Paleodontologia; Determinação da Idade pelos Dentes; Índios Sul-Americanos

\section{REFERÊNCIAS BIBLIOGRÁFICAS}

HILLSON, S., 1990. Teeth. Cambridge M anuals in Archaeology. Cambridge: Cambridge University Press.

JOHNSTON, F. E. \& ZIMMER, L. O., 1989. Assesment of growth and age in the imature skeleton. In: Reconstruction of Life From the Skeleton (M. Y. Iscan \& A. R. Kennedy, eds.), pp. 11-23, New York: Alan R. Liss.

ALVIM, M. C. M., 1977. Os antigos habitantes da área arqueológica de Lagoa Santa, Minas Gerais, Brasil - Estudos morfológicos. A rquivos do Museu de História Natural da UFMG, 2: 119173.

SOUZA, S. M. F. M., s/d. Paleopatologia humana de Santana do Riacho. Arquivos do Museu de História Natural da UFMG, 13. (No Prelo)

SOUZA, S. M. F. M. \& ALVIM, M. C. M., s/d. Estudo paleodemográfico da população humana do Grande Abrigo de Santana do Riacho, Minas Gerais. Arquivos do Museu de História Natural da UFMG, 13. (No Prelo)

OWSLEY, D. W. \& JANTZ, R. L., 1983. Formation of the permanent dentition in Arikara indians: timing differences that affect dental age assessments. American Journal of Physical A nthropology, 61: 467-471.

PROUS, A., 1989. Os sepultamentos da sondagem $n^{\circ}$ 1 do Grande Abrigo de Santana do Riacho. Relatórios das Escavações Arqueológicas. Belo Horizonte: Setor de Arqueologia, Museu de História Natural da Universidade Federal de Minas Gerais.

RADICHI, R., 1986. Estudo dos dentes, maxilas e mandíbulas dos restos esqueletais do sítio arqueológico de Santana do Riacho: determinação de idade pelo método radiográfico. Relatório de Análise de Esqueletos Humanos. Belo Horizonte: Setor de Arqueologia, Universidade Federal de Minas Gerais.

STEELE, D. G. \& BRAMBLET, C. A., 1988. The A natomy and Biology of the Human Skeleton. Texas: Texas A \& M University Press.

UBELAKER, D. H., 1978. Human Skeletal Remains: Excavation, Analysis, Interpretation. Chicago: Aldine.

WRIGHT, G. P. \& SYMMERS, W. S. T. C., 1966. Systemic Pathology. Vol. 2, London: Longmans. 